\title{
崇明东滩盐沼植被扩散格局及其形成机制
}

\author{
曹浩冰, 葛振鸣, 祝振昌, 张利权* \\ (华东师范大学河口海岸学国家重点实验室，上海 200062)
}

\begin{abstract}
摘要: 长江河口盐沼植被的形成和演化是生物与其生长环境相互作用的结果。以崇明东滩盐沼植被典型扩散前沿为研究对象, 2011 至 2012 年期间调查了盐沼植被扩散前沿实生苗扩散、定居以及形成的扩散格局, 同时测定了盐沼植被扩散前沿的潮滩冲 淤动态和水文动力条件。研究结果表明,崇明东滩盐沼植被在扩散前沿形成了互花米草-光滩 (Spartina alterniflora -Mudflat, SM) 和互花米草-海三棱薦草-光滩 (Spartina alterniflora-Scirpus mariqueter-Mudflat, SSM) 两种典型的扩散格局。冲淤动态和水文 动力条件是影响盐沼植被扩散格局的重要因子, 尤其是在 4-6 月盐沼植物实生苗传播和定居的关键阶段。在此基础上,分析 了东滩盐沼植被扩散前沿的生物-物理相互作用以及盐沼植被扩散格局的形成机制。研究结果不仅有助于理解长江河口地区 盐沼植被扩散的生物物理过程, 并对全球气候变化和海平面上升条件下滨海生态系统动态预测与湿地保护与管理具有重要的 意义。

关键词: 崇明东滩;盐沼植被;扩散格局;冲淤动态;水文动力条件;生物-物理相互作用

\section{The expansion pattern of saltmarshes at Chongming Dongtan and its underlying mechanism}

CAO Haobing, GE Zhenming, ZHU Zhenchang, ZHANG Liquan *

State Key Laboratory of Estuarine and Coastal Research, East China Normal University, Shanghai 200062, China

\begin{abstract}
The range expansion of saltmarshes in the Yangtze Estuary depends on accretion of dynamic mudflats resulting from the large amount of silt brought down the Yangtze River. The formation and succession of saltmarsh vegetation is a result of the interaction between saltmarsh plants and their living environment. Over the last 15 years, rapid invasion of large areas of the saltmarshes of Chongming Dongtan by an exotic plant Spartina alterniflora has occurred. Two types of advancing fronts are found: the S. alterniflora-mudflat (SM) front and the S. alterniflora-Scirpus mariqueter -mudflat ( SSM) front. The dynamic and natural characteristics of the coastal wetlands at Chongming Dongtan, both in terms of sediment deposition, salt marsh succession and biological invasion, make it an ideal place for studying the interaction between the range expansion of saltmarsh vegetation and the hydrodynamic regimes. In this study, we invested the seedling dispersal, establishment and formation of expansion pattern at the advancing fronts during the years of 2011 and 2012. At the same time, the accretion/erosion regime and hydrodynamic conditions at the saltmarsh expansion fronts were measured. The research aims were to: 1) test whether and how the hydrodynamic regimes govern the spatial structure of range expansion of saltmarshes at these two advancing fronts; 2) determine the interactions between the range expansion of saltmarsh and hydrodynamic regimes, and 3 ) understand the mechanisms underlying the range expansion of saltmarshes and their implications for the spatial structure of tidal saltmarshes. The results showed that the accretion/erosion regime and
\end{abstract}

基金项目:全球变化研究国家重大科学研究计划(2010CB951204); 国家自然科学基金(41201091); 上海市“浦江人才”计划( 13PJ1402200); 河 口海岸学国家重点实验室自主课题(44KZ001D)

收稿日期: 2013-04-11; 网络出版日期:2014-02-27

* 通讯作者 Corresponding author.E-mail: lqzhang@ sklec.ecnu.edu.cn 
hydrodynamic condition, especially in the critical stage of seedling establishment during April-June, were the most important factors responsible for the formation of these two typical expansion patterns. The rate of range expansion at the SM front was much faster than the SSM front. The different colonization behaviors at these two types of advancing fronts could be related to the differences in hydrodynamic regimes. At the site with a regime of autumn/winter erosion and spring/summer accretion, the sediment accretion and seedling recruitment of $S$. alterniflora were much higher, while the wave energy and current velocity were relatively gentle during the critical period of seedling establishment. At such site, the original pioneer species of $S$. mariqueter was replaced by S. alterniflora and a SM pattern of range expansion developed. In contrast, at the site with a relatively stable autumn/winter accretion regime and relatively strong wave energy and current velocity during the critical period of seedling establishment, the original pioneer species of $S$. mariqueter could remain within the advancing front and a SSM pattern of range expansion developed. Based on these results, the impact of abiotic and biotic factors governing the range expansion of and its implications on the spatial structure of tidal saltmarshes are discussed. The results from this study can provide a valuable insight into the bio-physical interactions on the formation of expansion patterns and the mechanisms underlying the range expansion of saltmarshes in the Yangtze Estuary, which could be important for the maintenance of coastal saltmarsh resource and biodiversity as well as the dynamics prediction of coastal saltmarshes under the impact of climate change.

Key Words: Chongming Dongtan; saltmarsh vegetation; expansion patterns; sedimentation dynamics; hydrodynamic condition ; bio-physical interaction

盐沼湿地是分布于海陆交界处受到波浪、潮汐、 沉积等多种复杂生物物理过程控制并具有多种生态 功能的典型生态系统 ${ }^{[1-2]}$ 。盐沼湿地面临着日益增 强的人类活动的干扰和破坏, 同时全球气候变化引 起的海平面上升也将进一步威胁盐沼湿地的丧 失 $^{[3]}$ 。许多研究表明, 盐沼植被的分布与动态是生 物与水动力、沉积等物理因素间相互作用的结 果 ${ }^{[4-6]}$ 。滨海湿地环境中的沉积、动力、地貌条件 (如 潮汐、波浪和沉积等) 可直接影响盐沼植被生活史中 的关键阶段 (如扩散、定居、生长和繁殖)，同时盐沼 植被也通过自身对水动力条件和沉积环境产生影 响 ${ }^{[7-11]}$ 。研究盐沼湿地分布与动态及其生物 -物理 相互作用与过程对盐沼湿地科学研究与湿地保护具 有重要的理论意义与科学价值 ${ }^{[4-6]}$ 。

长江口崇明东滩属于淤涨型滨海湿地, 盐沼植 被也处于动态变化中, 是研究盐沼植被时空格局与 生物-物理相互作用的理想环境 ${ }^{[4,11-12]}$ 。本项研究以 崇明东滩盐沼湿地为对象,在 2011 和 2012 年期间, 调查分析盐沼植被典型扩散前沿的扩散格局, 同时 测定扩散前沿的潮滩冲淤动态和水文动力条件，以 期探讨盐沼植被扩散格局与生物-物理相互作用及 其形成机制。研究结果不仅有助理解长江河口地区 盐沼植被扩散过程与机制, 而且对全球气候变化下
滨海湿地动态预测以及盐沼湿地保护具有着重要的 意义。

\section{1 材料和方法}

\section{1 研究区域概况}

崇明东滩湿地位于长江口崇明岛面向东海一 侧,包括 $31^{\circ} 25^{\prime}-31^{\circ} 38^{\prime} \mathrm{N}, 121^{\circ} 50^{\prime}-122^{\circ} 05^{\prime} \mathrm{E}$ 之间 的大部分区域 (图 1)。该地区地处北亚热带,气候 温和湿润, 四季分明,夏季湿热, 盛行东南风, 冬季干 冷, 盛行偏北风, 属典型的季风气候。年平均气温为 $15.3{ }^{\circ} \mathrm{C}$, 月平均气温以 1 月的 $2.8{ }^{\circ} \mathrm{C}$ 为最低, 以 7 月 的 $27.5{ }^{\circ} \mathrm{C}$ 为最高。降水主要集中在每年的 4-9 月, 年平均降水量 $1022 \mathrm{~mm}$, 相对湿度 $82 \%^{[13]}$ 。受到非 正规半日潮的作用, 其每日潮汐有昼夜两次变化, 平 均潮差 $2.66 \mathrm{~m}$ 。长江大量来沙不断在此淤积, 使崇 明东滩滨海湿地每年向外延伸 $150-300 \mathrm{~m}^{[11,14]}$ 。 近年来随着长江上游三峡工程等修建, 导致长江来 沙量锐减, 其淤积速率逐渐减缓 ${ }^{[15-16]}$ 。

在互花米草 (Spartina alterniflora)引人崇明东滩 以前, 崇明东滩盐沼植被分布和演替呈带状趋势, 在 高程低于 $2 \mathrm{~m}$ 的中潮滩下缘和低潮滩的盐渍藻类带 (光滩) 区域无高等植物分布, 高程位于 $2-2.9 \mathrm{~m}$ 的 中潮滩上部和高潮滩为海三棱蔍草 (Scirpus 
mariqueter) 和蔍草 (Scirpus triqueter) 带, 高程一般在 $2.8 \mathrm{~m}$ 以上的高潮滩上部则为芦苇 (Phragmites australis) 带, 此外此外还散生着一些白茅 (Imperata cylindrica)、碱蓬 (Suaeda glauca)、糙叶苔草 (Carex scabrifolia) 和野灯芯草 (Juncus setchuensis) 等玨块状 群落。自 1995 年互花米草被引人崇明东滩, 互花米 草逐渐在东滩定居, 通过其极强的有性和无性繁殖 进行快速扩散。目前互花米草群落以连续锋面状扩 散模式形成了大面积单优群落, 其分布下限可达到 海三棱蔍草带, 对本地原生植被芦苇和海三棱蔍草
形成了强烈的竞争态势 ${ }^{[11,17]}$ 。

前期的研究表明, 崇明东滩盐沼植被前缘地带 主要有两种典型的扩散格局, 一是互花米草-光滩 (Spartina alterniflora-Mudflat) 前沿 (以下简称 SM), 二是互花米草-海三棱蔍草-光滩 (Spartina alternifloraScirpus mariqueter-Mudflat) 前沿 (以下简称 SSM) ${ }^{[11-12,18]}$ 。 2011 至 2012 年期间, 选择崇明东滩自然保护区北部 试验区典型的盐沼植被 SM 前沿( $31^{\circ} 35^{\prime} 50.4^{\prime \prime}, 121^{\circ}$ 53 '25.0") 和 SSM 前沿 $\left(31^{\circ} 34^{\prime} 48.5^{\prime \prime}, 121^{\circ} 54^{\prime}\right.$ 52. $6^{\prime \prime}$ ) 为研究区域(图 1)。

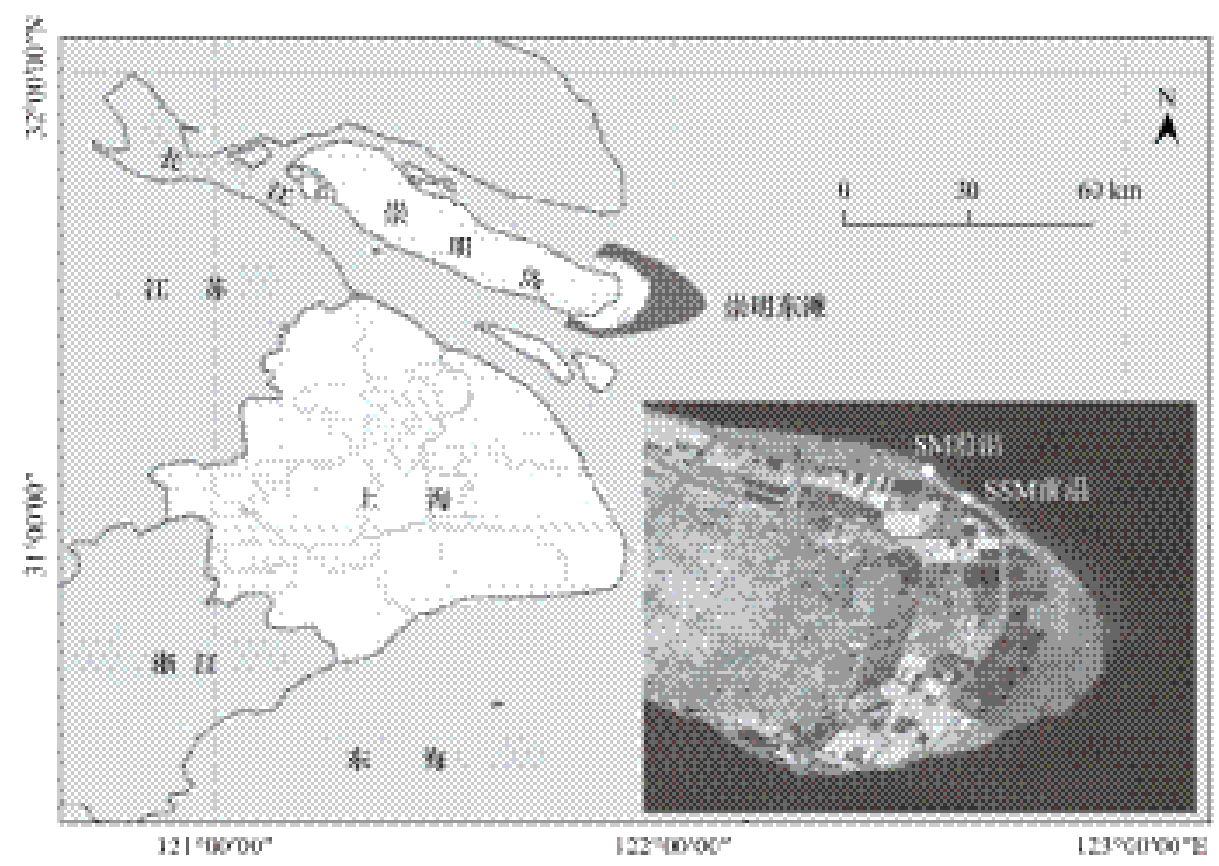

图 1 崇明东滩及研究区域示意图 (SM 前沿为互花米草-光滩前沿,SSM 前沿为互花米草-海三棱蔍草-光滩前沿)

Fig.1 Location of Chongming Dongtan and the study area, SM front represents Spartina alterniflora-Mudflat front and SSM front represents Spartina alterniflora-Scirpus mariqueter-Mudflat front

\section{2 盐沼植被扩散动态与格局调查}

于 2011 年 3 月, 分别在 SM 和 SSM 扩散前沿的 连续植被带 (定义为植被盖度> $50 \%$ ), 向海方向的光 滩上设置 $150 \mathrm{~m}$ 长与 $50 \mathrm{~m}$ 宽的研究区域 (图 2)。 2011 年 5 月, 沿向海方向每间隔 $25 \mathrm{~m}$ 随机选取 10 个 $1 \mathrm{~m} \times 1 \mathrm{~m}$ 的样方, 调查记录 SM 和 SSM 扩散前沿 的实生苗密度。2012 年的研究区域相应前移至 2011 年扩散形成的连续植被带前沿。沿样带从连续 植被带边缘向海方向, 每间隔 $25 \mathrm{~m}$ 设置 5 个 $1 \mathrm{~m} \times 1$ $\mathrm{m}$ 的固定样方 (每样方间距 $10 \mathrm{~m}$ 左右), 各 30 个 (图 2 )。2012 年 4-6 月, 调查记录固定样方中实生苗密 度与存活。至 10 月生长季末期, 在固定样方内随机 选取 10 植株, 测定其株高和分藮数。
2011 年和 2012 年, 分别在 SM 和 SSM 扩散前沿 的连续植被带边缘向海方向, 各设立 1 个 $90 \mathrm{~m} \times 15 \mathrm{~m}$ 以木桩标记的格局监测样区 (图 2)。同样, 2012 年 的格局监测样区前移至 2011 年扩散形成的连续植 被带前沿。用方格纸调查记录两种前沿在生长季始 (3月) 和生长季末 (10月) 的扩散格局。每个方格 代表 $1 \mathrm{~m} \times 1 \mathrm{~m}$ 的空间网格，网格数据录人 EXCEL2007 软件后分析 2011 年和 2012 年盐沼植被 扩散前沿的时空格局。

1.3 盐沼植被前沿冲淤动态与水文动力条件测定 盐沼植被前沿冲淤动态监测采用标记桩法。 2010 年 11 月分别在 SM 和 SSM 扩散前沿的研究区 域内设置 3 条样线 (间距 $10 \mathrm{~m}$ 左右), 从连续植被带 
边缘向海方向沿样线每 $25 \mathrm{~m}(0 、 25 、 50 、 75 \mathrm{~m}$ 和 100 $\mathrm{m}$ ) 设置标记木桩图 2)。标记木桩 $1.5 \mathrm{~m}$ 长, 垂直打 人潮滩并露出 $40 \mathrm{~cm}$ 左右。每月监测标记桩顶端距 滩面垂直距离的变化,统计时以相对于初始高程变 化的平均值表达潮滩高程的冲淤动态。2011 年 11 月, 以相同方法前移至当年形成的连续植被带前沿, 继续监测 2012 年度扩散前沿潮滩高程的冲淤状况。

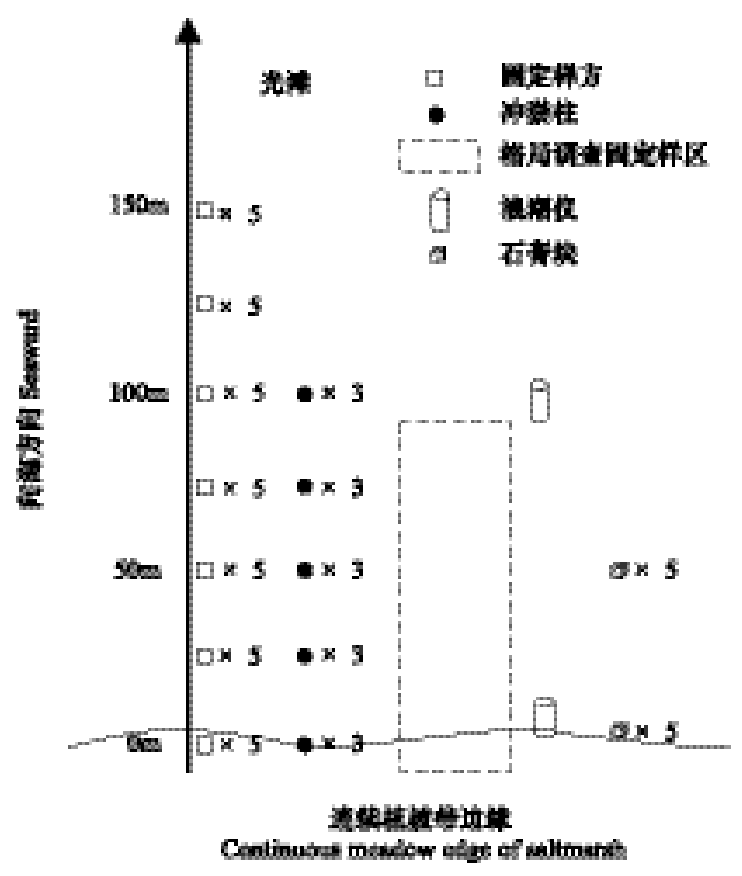

图 2 扩散前沿研究区域、样区、样线与样方设置示意图

Fig.2 Schematic distribution of the study area, monitoring block, sampling transects and plots at the expansion fronts

2011 年 4 月 17 - 22 日 (大-小潮周期), 利用 SBE 26plus 浪潮仪 ( SEA BIRD Electronics Inc., USA) 测定实生苗定居阶段的扩散前沿潮滩波浪强 度。监测时分别在 SM 和 SSM 前沿距连续植被带边 缘 $5 \mathrm{~m}$ 和 $100 \mathrm{~m}$ 处各放置 1 台浪潮仪( 图 2)。将浪 潮仪固定到专用架上并平放于滩面, 保持压力传感 器距滩面 $15 \mathrm{~cm}$, 设置采样时长间隔 $10 \mathrm{~min}$, 测量频 率 $4 \mathrm{~Hz}$ 。并检测了仪器的工作状态, 以保证浪潮仪 所测得数据的可靠性, 利用专用软件 SeasoftWaves 导 出和转化记录数据。记录数据参数包含平均波高、 有效波高、最大波高、波能密度等，本文选取波能密 度参数来比较 SM 和 SSM 扩散前沿的波浪强度。

在测定扩散前沿潮滩波浪强度同期,采用石膏 溶解法 ${ }^{[7]}$ 测定扩散前沿的潮滩水流速度。备好已称 量至恒重 $\left(M_{1}\right)$ 的 $7 \mathrm{~cm} \times 5 \mathrm{~cm} \times 5 \mathrm{~cm}$ 的石亳块, 用塑 料纸包裹留出 $5 \mathrm{~cm} \times 5 \mathrm{~cm}$ 的一面, 并将暴露面向上
固定于专门设置的木桩上 (距滩面约 $10 \mathrm{~cm}$ )。石膏 块分别设立在 SM 和 SSM 扩散前沿的 $5 \mathrm{~m}$ 和 $55 \mathrm{~m}$ 处,每一处设置 5 块, 间距 $3 \mathrm{~m}$ 左右 (图 2)。观测结 束后, 回收石膏块自然风干至恒重 $\left(M_{2}\right)$ 并计算石膏 块实验前后的质量损失 $\left(M L=M_{2}-M_{1}\right)$ 。根据石膏 块的质量损失以及石膏溶解质量与水流速度的正比 关系, 比较各观测点的水流速度 ${ }^{[19]}$ 。

\section{4 数据处理}

使用 SPSS18.0 统计分析软件对 SM 和 SSM 扩散 前沿的连续植被带扩散距离、冲淤动态和波浪强度 的监测结果进行独立样本 $t$ 检验。采用非参数 Mann-Whitney U 检验比较 SM 和 SSM 扩散前沿水流 速度 (石膏质量损失) 和实生苗定居、存活和生长数 据的差异性(显著性水平 0.05)。并用 EXCEL2007 和 Origin8.5 软件包作图。

\section{2 结果}

\section{1 盐沼植被扩散前沿实生苗定居动态}

崇明东滩盐沼植被前沿实生苗扩散的调查结果 表明, 2011 年 5 月末, SM 前沿的互花米草实生苗扩 散范围可达距连续植被带向海方向 $100 \mathrm{~m}$ 内的光 滩, 而 SSM 前沿的扩散距离为 $50 \mathrm{~m}$ 内。两种扩散前 沿在 $0-25 \mathrm{~m}$ 区域内实生苗密度最大, 而随着与连 续植被带距离增加而实生苗密度均减少。SM 前沿 实生苗密度显著高于 $\mathrm{SSM}$ 前沿 $(P<0.05)$, 而 SM 和 $\mathrm{SSM}$ 前沿扩散实生苗的存活率则基本相同, 分别为 92\% 和 91\% (表 1)。2012 年,SM 前沿的实生苗扩散 范围可达距连续植被带向海方向 $150 \mathrm{~m}$ 内的光滩, 而 SSM 前沿的实生苗扩散范围为 $75 \mathrm{~m}$ 内。至 6 月 中旬, SM 前沿实生苗密度显著高于 SSM 前沿 $(P<$ $0.05)$, 两种前沿实生苗密度亦随与连续植被带距离 增加而呈减少趋势。SM 前沿实生苗 4-5 月的存活 率分别为 3.8\% 和 70\%, 而该时段 SSM 前沿实生苗的 存活率为 0 , 但至 6 月中旬, 两种扩散前沿已定居实 生苗的存活率都可达 100\% (表 1)。

2012 年固定样方内实生苗生长的监测结果显 示, 至当年 10 月生长季末期, 定居于 SM 扩散前沿的 实生苗平均分藥数和平均株高分别为 $(19.0 \pm 7.5)$ 株 和 $(60.3 \pm 14.6) \mathrm{cm}$, 而定居于 SSM 扩散前沿的实生 苗平均分繤数和平均株高分别为 $(4.6 \pm 3.0)$ 株和 $(54.8 \pm 11.4) \mathrm{cm}$ (表 2)。其中定居于 SM 扩散前沿实 
生苗的平均分藥数显著高于 SSM 扩散前沿 $(P<$ 散前沿, 但两者无显著差异 $(P>0.05)$ ( 表 2)。 $0.05), \mathrm{SM}$ 扩散前沿植株的平均株高略高于 SSM 扩

表 12011 和 2012 年 SM 前沿 (互花米草-光滩前沿) 和 SSM 前沿 (互花米草-海三棱蔍草-光滩前沿) 实生苗的定居动态

Table 1 The dynamics of seedling establishment at the SM front (Spartina alterniflora -Mudflat front) and the SSM front (Spartina alternifloraScirpus mariqueter-Mudflat front) in 2011 and 2012

\begin{tabular}{|c|c|c|c|c|c|c|c|c|}
\hline \multirow{3}{*}{$\begin{array}{l}\text { 前沿 } \\
\text { Front }\end{array}$} & \multirow{3}{*}{$\begin{array}{l}\text { 时间 } \\
\text { Time }\end{array}$} & \multicolumn{6}{|c|}{$\begin{array}{c}\text { 实生苗密度 Seedling density }\left(\text { 株 } / \mathrm{m}^{2}\right)(\text { mean } \pm \text { S.D. }) \\
\text { 距连续植被带距离 Distance from the continuous meadow edge }\end{array}$} & \multirow{3}{*}{$\begin{array}{l}\text { 存活率/\% } \\
\text { Survivorship }\end{array}$} \\
\hline & & \multicolumn{6}{|c|}{ 距连续植被带距离 Distance from the continuous meadow edge } & \\
\hline & & $0-25 \mathrm{~m}$ & $25-50 \mathrm{~m}$ & $50-75 \mathrm{~m}$ & $75-100 \mathrm{~m}$ & $100-125 \mathrm{~m}$ & $125-150 \mathrm{~m}$ & \\
\hline 互花米草-光滩 & 2011-05-26 & $2.5 \pm 0.8$ & $1.6 \pm 0.7$ & $1.1 \pm 0.8$ & $0.7 \pm 0.5$ & 0 & 0 & 92 \\
\hline \multirow[t]{3}{*}{ S. alterniflora-Mudflat } & 2012-04- 10 & $0.4 \pm 0.8$ & $1.8 \pm 2.0$ & $3.4 \pm 3.2$ & $0.2 \pm 0.4$ & 0 & 0 & 3.8 \\
\hline & 2012-05-13 & $1 \pm 1.7$ & $0.2 \pm 0.4$ & $0.6 \pm 0.8$ & 0 & 0 & $0.2 \pm 0.4$ & 70 \\
\hline & 2012-06- 17 & $3.0 \pm 1.6$ & $1.2 \pm 1.3$ & $0.2 \pm 0.4$ & 0 & 0 & $0.2 \pm 0.4$ & 100 \\
\hline 互花米草- & 2011-05-26 & $1.3 \pm 1.3$ & $0.7 \pm 0.8$ & 0 & 0 & 0 & 0 & 91 \\
\hline 海三棱蔍草-光滩 & 2012-04- 10 & $0.6 \pm 0.8$ & $2.4 \pm 2.0$ & $1.2 \pm 1.3$ & 0 & 0 & 0 & 0 \\
\hline S. alterniflora- & $2012-05-13$ & 0 & $0.2 \pm 0.4$ & 0 & 0 & 0 & 0 & 0 \\
\hline S. mariqueter-Mudflat & 2012-06- 17 & $0.6 \pm 1.3$ & $0.2 \pm 0.4$ & $0.8 \pm 1.3$ & 0 & 0 & 0 & 100 \\
\hline
\end{tabular}

表 2 SM 和 SSM 扩散前沿中 2012 年生长季末定居实生苗的生长状 况 (平均值土标准差)

Table 2 Growth of the established seedlings at the SM and SSM fronts in the end of growing season of $2012(\operatorname{mean} \pm$ S.D. $)$

\begin{tabular}{ccc}
\hline $\begin{array}{c}\text { 扩散 } \\
\text { Expansion front }\end{array}$ & $\begin{array}{c}\text { 分藥数 } \\
\text { Tillers (株/株苗 }\end{array}$ & $\begin{array}{c}\text { 株高 } \\
\text { Height } / \mathrm{cm}\end{array}$ \\
\hline SM & $19 \pm 7.5$ & $60.3 \pm 14.6$ \\
SSM & $4.6 \pm 3.0$ & $54.8 \pm 11.4$ \\
\hline
\end{tabular}

\section{2 盐沼植被前沿扩散格局}

图 3 显示了崇明东滩盐沼植被 SM 和 SSM 扩散 前沿的时空格局。从 2011 年度和 2012 年度的 3 月 生长季开始,大量的互花米草实生苗定居于 SM 前沿 的光滩。成功定居的实生苗迅速生长和分菜,形成 互花米草斑块并通过斑块扩大和融合, 至当年生长 季末 10 月形成新的连续植被带。按植被盖度> $50 \%$ 的定义,SM 前沿连续植被带 2011 年度向前扩展了 $(58.9 \pm 0.9) \mathrm{m}, 2012$ 年度向前扩展了 $(56 \pm 1.2) \mathrm{m}$ 。而 在 SSM 前沿定居的互花米草实生苗很少, 主要是通 过海三棱蔍草群落锋面状向前扩展。2011 年度和 2012 年度, SSM 前沿分别向前仅扩展了 $(11.2 \pm 2.1)$ $\mathrm{m}$ 和 $(6.7 \pm 3.1$ ) $\mathrm{m}$ (图 3)。SM 前沿连续植被带 2011 年度和 2012 年度的扩展距离均显著高于 SSM 前沿 $(P<0.05)$, 而同一前沿其扩展距离的年际差异不显 著 $(P>0.05)$ 。

\section{3 盐沼植被前沿冲淤动态与水文动力条件}

\subsection{1 潮滩冲淤动态}

崇明东滩盐沼植被扩散前沿潮滩冲淤动态监测
结果表明,SM 前沿潮滩在 2010 年 11 月至 2011 年 3

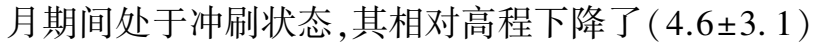
$\mathrm{cm}$,而从 2011 年 4 月开始淤积抬升, 至 2011 年 10 月其相对高程累计淤积 $(19.3 \pm 7.4) \mathrm{cm}$ (图 4)。而 SSM 前沿在 2010 年 11 月至 2011 年 2 月期间其滩面 高程无显著变化, 从 2011 年 3 月开始淤积抬升, 至 2011 年 10 月其相对高程累计淤积 $(12.3 \pm 4.5) \mathrm{cm}_{\text {。 }}$ 2011 年度,SM 前沿潮滩相对高程累计淤积显著高于 SSM 扩散前沿 $(P<0.05)$ 。

2012 年度, SM 和 SSM 前沿潮滩冲淤动态基本 与 2011 年度相似 (图 4)。SM 前沿潮滩在 2011 年 12 月至 2012 年 3 月期间处于冲刷状态, 其相对高程 下降了 $(3.1 \pm 1.3) \mathrm{cm}$, 而从 2012 年 4 月开始淤积抬 升, 至 2011 年 11 月其相对高程累计淤积 (16.6土

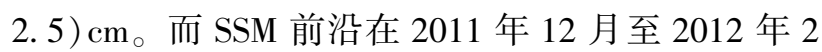
月期间其滩面高程无显著变化,从 2012 年 3 月开始 淤积抬升, 至 2012 年 11 月其相对高程累计淤积 (11.4 44.2$) \mathrm{cm}$ (图 4)。

\subsection{2 盐沼植被前沿波浪强度和流速}

盐沼植被扩散前沿在实生苗定居关键期 (4 月 17-22 日)潮滩波能密度测定结果显示, SM 前沿距 连续植被带 $5 \mathrm{~m}$ 和 $100 \mathrm{~m}$ 处的平均波能密度分别为 $23.6 \mathrm{~J} / \mathrm{m}^{2}$ 和 $30.8 \mathrm{~J} / \mathrm{m}^{2}$, 最大波能密度分别为 222.2 $\mathrm{J} / \mathrm{m}^{2}$ 和 $211.2 \mathrm{~J} / \mathrm{m}^{2}$ 。而 $\mathrm{SSM}$ 前沿 $5 \mathrm{~m}$ 和 $100 \mathrm{~m}$ 处的 平均波能密度分别为 $35.2 \mathrm{~J} / \mathrm{m}^{2}$ 和 $43.1 \mathrm{~J} / \mathrm{m}^{2}$, 最大波 能密度分别为 $249.3 \mathrm{~J} / \mathrm{m}^{2}$ 和 $285.8 \mathrm{~J} / \mathrm{m}^{2}$ (图 5 )。 $\mathrm{SM}$ 
前沿的波能密度显著低于 SSM 前沿 $(P<0.05)$, 说明

该期间 SM 前沿的水文动力条件弱于 SSM 前沿。
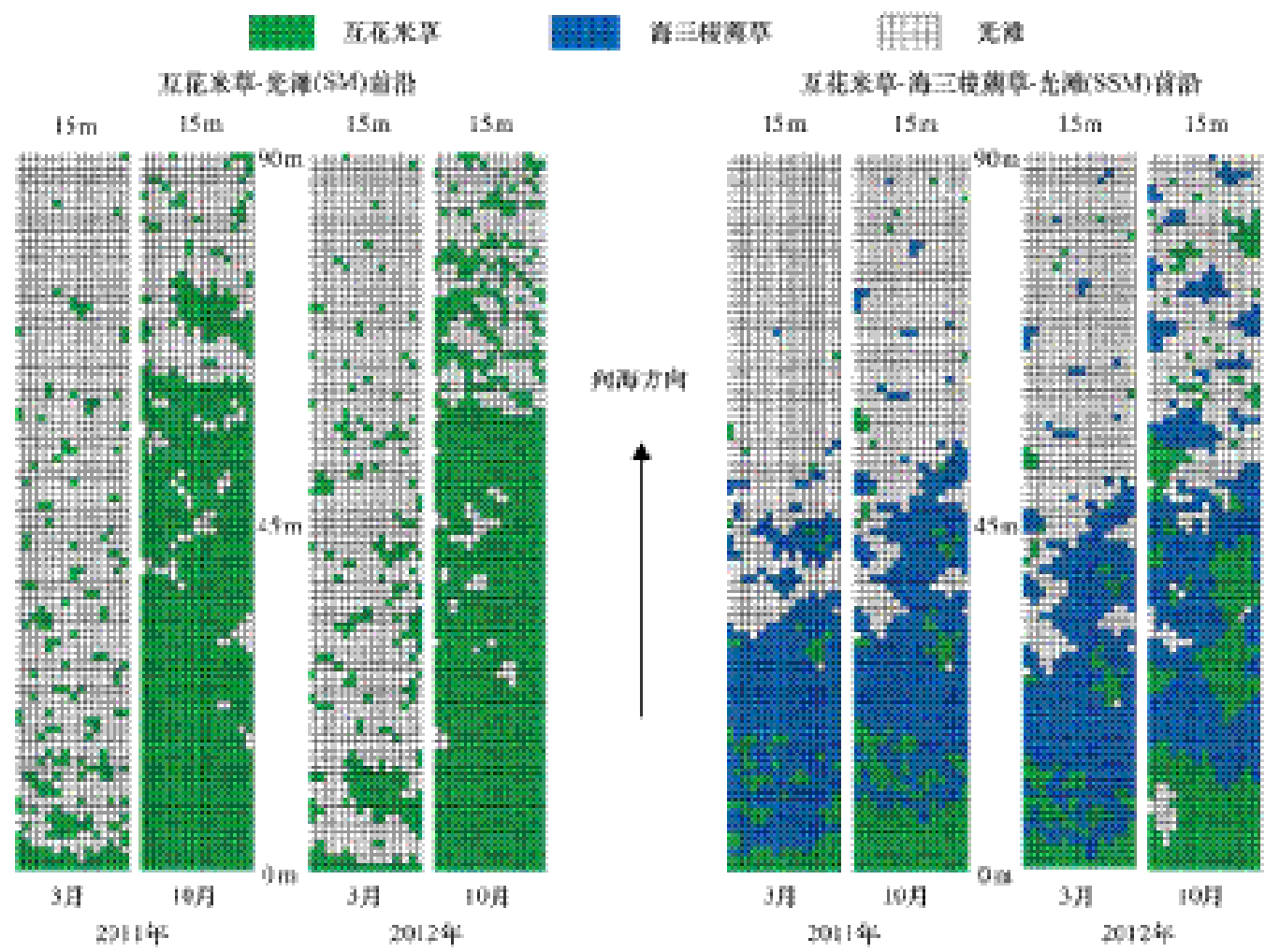

图 3 盐沼植被 SM 和 SSM 前沿在 2011 和 2012 年度的扩散格局

Fig.3 The expansion patterns of saltmarsh vegetation at the SM and SSMfronts in 2011 and 2012

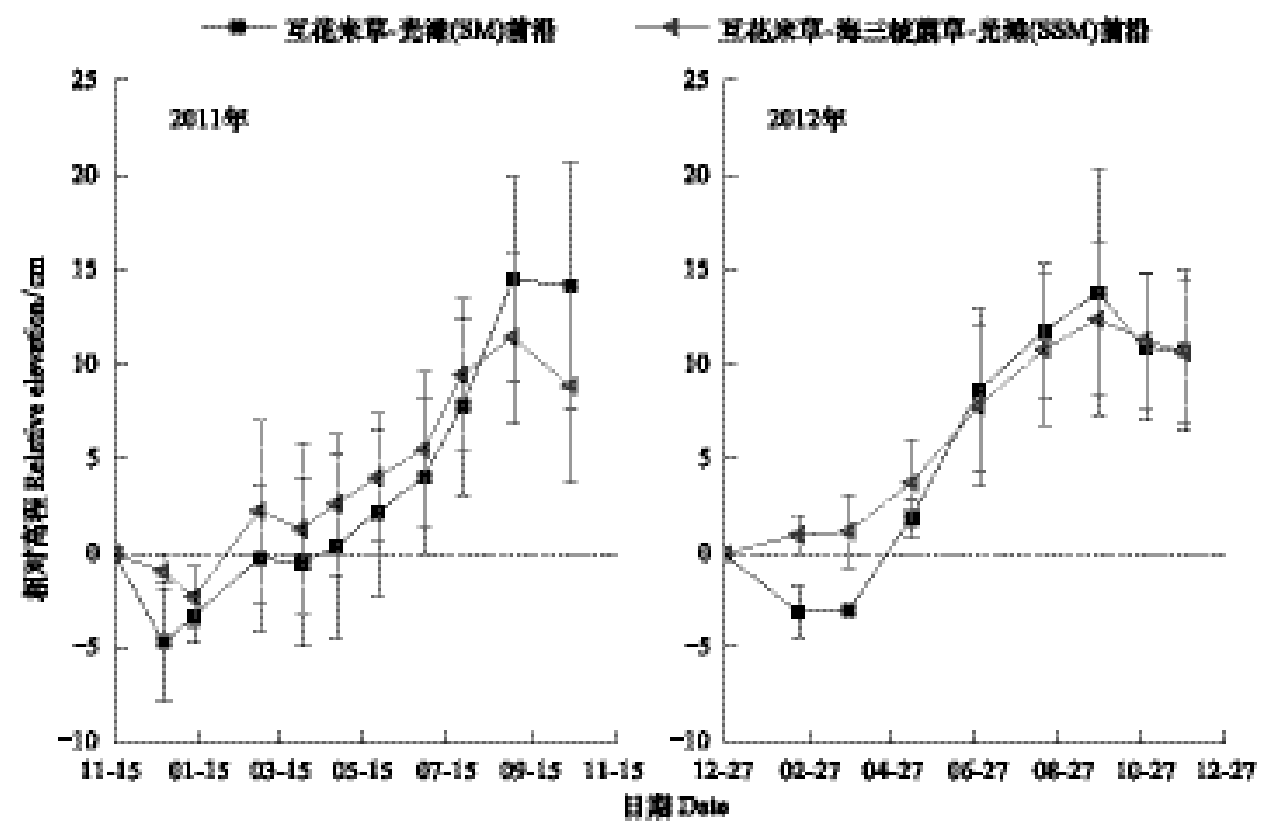

图 42011 年度和 2012 年度 SM 和 SSM 前沿的冲淤动态

Fig.4 The accretion/erosion dynamics at the SM and SSM fronts in 2011 and 2012

同时期,盐沼植被扩散前沿的水流速度测定结 果显示, $\mathrm{SM}$ 前沿距连续植被带 $5 \mathrm{~m}$ 和 $55 \mathrm{~m}$ 处的石
膏块平均质量损失分别为 $(4.0 \pm 1.2) \mathrm{g}$ 和 $(3.3 \pm 1.7)$ $\mathrm{g}$, 而 SSM 前沿距连续植被带 $5 \mathrm{~m}$ 和 $55 \mathrm{~m}$ 处的石膏 
块平均质量损失分别为 $(7.0 \pm 0.7) \mathrm{g}$ 和 $(5.7 \pm 1.9) \mathrm{g}$ 前沿 $(P<0.05)$, 也说明了在实生苗定居关键期 (4 月 (图 6)。SM 前沿的石膏块质量损失显著低于 SSM 17-22 日) SM 前沿的水文动力条件弱于 SSM 前沿。

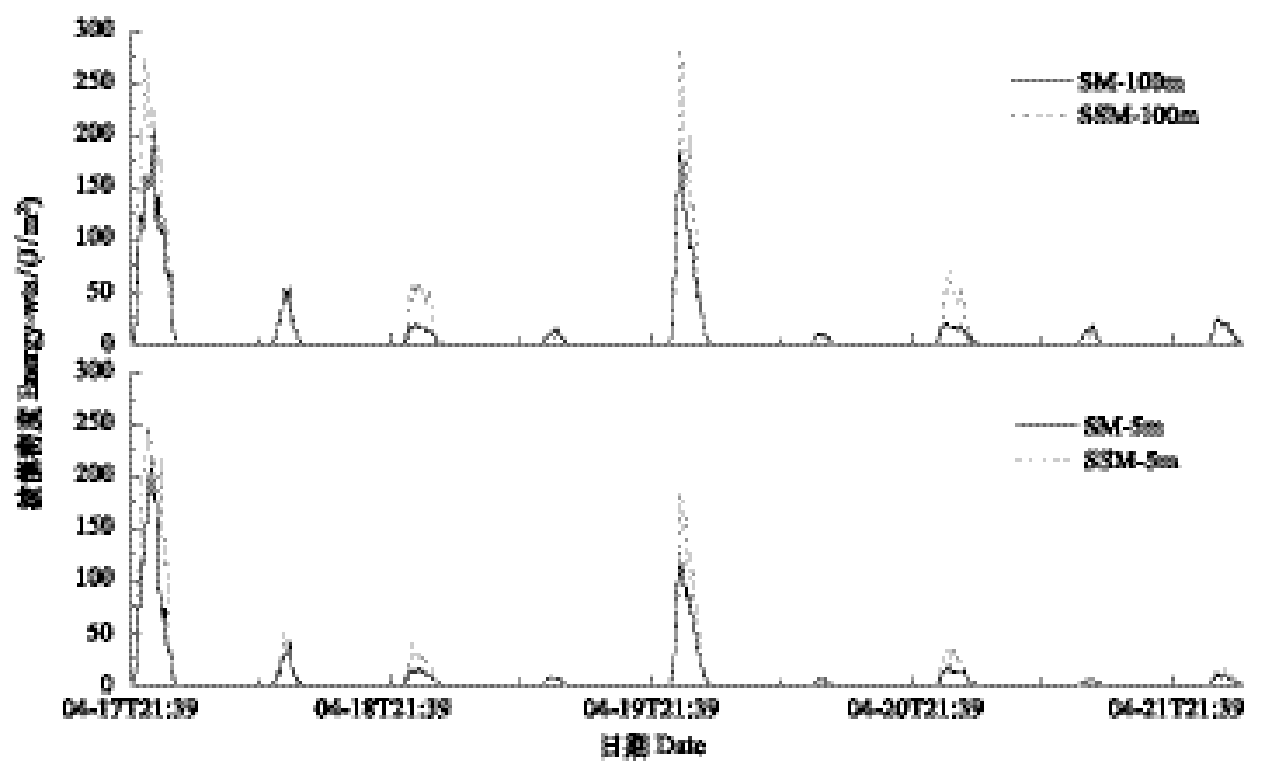

图 52011 年 4 月观测期间 SM 和 SSM 前沿的波能密度

Fig.5 The wave energy at the SM and SSM fronts during the observation period of April, 2011

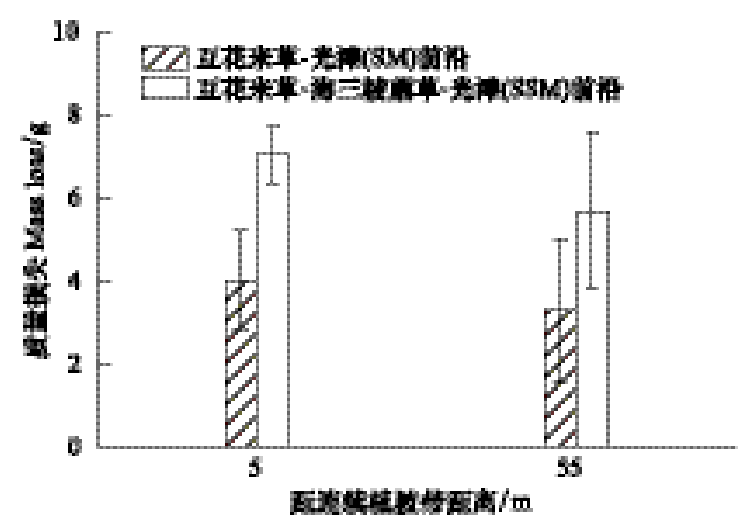

图 62011 年 4 月观测期间 SM 和 SSM 前沿的石亳质量损失 Fig.6 Mass loss of the plaster blocks at the SM and SSM fronts during the observation period of April, 2011

\section{3 讨论}

崇明东滩为淤涨型河口湿地, 长江来沙在此不 断淤积促进了潮滩的淤涨, 而不断淤涨的东滩湿地 为盐沼植被的扩展提供了大量的新生生境 ${ }^{[12,14]}$ 。 2011-2012 年度崇明东滩盐沼植被前沿扩散格局的 研究表明,每年 3 月生长季开始,大量的互花米草实 生苗通过潮汐运动定居于 $\mathrm{SM}$ (互花米草-光滩) 前沿 的光滩。成功定居的实生苗迅速生长和克隆 (分 薬), 形成互花米草斑块并通过斑块扩大和融合, 至 当年生长季末形成新的 SM 前沿。SM 前沿互花米草
扩散格局与美国 Willapa 湾互花米草盐沼的扩散格 局相同 ${ }^{[20]}$ 。而在 $\operatorname{SSM}$ (互花米草-海三棱蔍草-光滩) 前沿, 互花米草实生苗定居很少, 主要是通过海三棱 蔍草群落锋面状向前缓慢扩展, 至当年生长季末形 成新的 SSM 扩散前沿。2011-2012 年崇明东滩盐 沼植被 SM 和 SSM 扩散前沿的时空格局与 20082010 年在同一地点研究结果一致 ${ }^{[11-12]}$, 表明崇明东 滩盐沼植被已形成这两种典型和相对稳定的扩散 模式。

许多研究表明, 河口湿地环境中的沉积动力条 件 (如潮汐、波浪和沉积等) 直接影响盐沼植被的生 长和分布。江苏地区互花米草盐沼动态研究表明, 盐沼植被动态与沉积动力条件关系密切, 在淤积速 率较高的区域,其互花米草盐沼扩展广阔,而在淤积 速率较低的区域,互花米草盐沼植被扩展缓慢甚至 退化 ${ }^{[21]}$ 。有关崇明东滩潮间带沉积动力地貌的研 究表明,崇明东滩为淤涨型河口湿地, 具明显的季节 性冲淤动态, 总体上呈现出秋冬侵蚀与春夏淤涨的 特征,但在不同区域和季节也存在差异 ${ }^{[22-23]}$ 。本项 研究结果表明, SM 前沿呈现出秋冬季冲刷、春夏季 快速淤积的冲淤动态, 而 SSM 前沿呈现秋冬季其潮 滩相对稳定, 春夏季淤积的冲淤动态 (图 4)。在春 夏季潮滩淤积的过程中, SM 前沿净淤积量 (高度和 
淤涨范围) 显著高于 SSM 前沿,相应的 SM 前沿盐沼 植被扩散距离也显著大于 SSM 前沿(图 3)。崇明东 滩潮滩冲淤动态与潮间带水动力条件关系密 切 ${ }^{[22-23]}$ 。SM 前沿秋冬季潮滩的冲刷表明该时段其 水动力条件强于秋冬季潮滩相对稳定的 SSM 前沿。 互花米草为多年生草本, 植株粗壮高大, 具有发达的 根茎, 其抗冲刷能力强, 而海三棱蔍草植株低矮, 根 茎较弱, 其抗冲刷能力弱 ${ }^{[11]}$ 。因此, 秋冬季潮滩冲 刷区域有利于互花米草取代海三棱蔍草形成 SM 前 沿,而秋冬季潮滩相对稳定区域则有利于海三棱蔍 草生存而形成 SSM 前沿。

在盐沼植物生活史中的关键阶段如扩散、定居、 生长和繁殖, 河口湿地环境中的沉积动力条件对盐 沼植被扩散的影响尤为关键 ${ }^{[7,10,24-25]}$ 。崇明东滩盐 沼植被快速扩散主要依赖于盐沼植物的繁殖体传播 和定居 ${ }^{[11-12]}$ 。在盐沼植被快速扩散的关键阶段 (46 月), SM 和 SSM 前沿都表现为淤积状态 (图 4), 但 SM 前沿的淤积量显著高于 SSM 前沿。该时期的水 文动力条件 (波能密度和流速) 测定也表明了 SM 前 沿的水文动力条件弱于 SSM 前沿(图 5 和图 6)。 2010 年春夏季淤积期在同一地点 SM 和 SSM 前沿的 水文动力条件测定也呈现了相同的趋势 ${ }^{[18]}$ 。同时 期同一地点沉积物组成调查结果显示, SM 前沿和 SSM 前沿沉积物的物理组成基本相同,其沉积物粒 径分别为 $d_{50}=(6.46 \pm 0.24) \mu \mathrm{m}$ 和 $(8.83 \pm 0.66) \mu \mathrm{m}$, 沉积物中粘土比例均超过 $7.5 \%$ 的阈值 ${ }^{[18]}$, 可以说明 沉积物物理组成并不是两种前沿实生苗定居出现差 异的主要原因。在春夏季快速淤涨的 SM 前沿,其较 适宜的沉积动力条件有利于互花米草实生苗快速扩 散和定居,并取代海三棱蔍草而形成 SM 扩散格局。 而 SSM 前沿在春夏季淤涨期的较强水动力条件则不 利于互花米草实生苗的定居,进而形成 SSM 扩散格 局。荷兰 Westerschelde 河口盐沼中大米草 (Spartina anglica) 扩散和定居的研究结果也表明,在强水动力 条件下, 大米草实生苗的定居和存活存在个体生物 量阈值, 其生物量低于阈值则难以定居和存活 ${ }^{[4]}$ 。

许多研究表明,盐沼植被通过自身也会对潮滩 沉积动力条件产生影响 ${ }^{[4,5,18,25-26]}$ 。无论在原产地还 是人侵地, 互花米草对潮滩的非生物环境可以产生 明显的作用, 被认为是生态系统工程师 ${ }^{[27]}$ 。互花米 草高大密实的植被可以减弱水动力条件并促进沉积
作用 ${ }^{[10,25]}$, 而改变后的沉积动力环境又促进了互花 米草的定居和扩散,形成盐沼植被与沉积动力环境 的正反馈环路 ${ }^{27]}$ 。美国 Willapa 湾的研究表明, 互 花米草盐沼可以降低水流速度并促进淤积速率, 从 而提高了其实生苗的定居和克隆生长, 加快了互花 米草群落的扩散速率 ${ }^{[28]}$ 。崇明东滩盐沼植被 SM 前 沿互花米草群落与潮滩沉积动力条件的相互作用也 表现为这种正反馈机制。

崇明东滩作为淤涨型河口湿地, 长江来沙不断 在此淤积促进了潮滩扩展,盐沼植被时空格局也处 于动态变化过程中。盐沼植被与潮滩沉积动力条件 的相互作用,形成了与其环境条件相适应的扩散格 局。本项研究对这些过程和作用的探讨有利于深人 崇明东滩盐沼植被分布和扩散格局形成机制。这不 仅有助于理解整个长江河口地区盐沼植被的生物物理过程,还将对全球气候变化和海平面上升条件 下滨海湿地动态预测 , 以及相应湿地保护和管理措 施的制定具有重要意义。

\section{References :}

[ 1 ] Tyler A C, Zieman J C. Patterns of development in the creekbank region of a barrier island Spartina alterniflora marsh. Marine Ecology Process Series, 1999, 180: 161-177.

[ 2 ] Davidson-Arnott R G D, van Proosdij D, Ollerhead J, Schostak L. Hydrodynamics and sedimentation in salt marshes: examples from a macrotidal marsh, Bay of Fundy. Geomorphology, 2002, 48(1/3) : 209-231.

[ 3 ] Gedan K B, Silliman B R, Bertness M D. Centuries of humandriven change in salt marsh ecosystems. Annual Review of Marine Science, 2009, 1(1): 117-141

[ 4 ] van Wesenbeeck B K, van de Koppel J, Herman P M J, Bouma T J. Does scale-dependent feedback explain spatial complexity in salt-marsh ecosystems?. Oikos, 2008, 117(1) : 152-159.

[ 5 ] van Wesenbeeck B K, van de Koppel J, Herman P M J, Bakker J $\mathrm{P}$, Bouma T J. Biomechanical warfare in ecology; negative interactions between species by habitat modification. Oikos, 2007, $116(5): 742-750$.

[ 6 ] Callaghan D P, Bouma T J, Klaassen P, van der Wal D, Stive M J F, Herman P M J. Hydrodynamic forcing on salt-marsh development: Distinguishing the relative importance of waves and tidal flows. Estuarine, Coastal and Shelf Science, 2010, 89(1): 73-88.

[ 7 ] Bruno J F. Facilitation of cobble beach plant communities through habitat modification by Spatina alterniflora. Ecology, 2000, 81 (5) : 1179-1192. 
[ 8 ] Ehrenfeld J G. Dynamics and processes of barrier island vegetation. Reviews in Aquatic Sciences, 1990, 2 ( 3/4): 437- 480 .

[ 9 ] Bertness M D. Zonation of Spartina patens and Spartina alterniflora in New England salt marsh. Ecology, 1991, 72(1): 138-148.

[10] Rand T A. Seed dispersal, habitat suitability and the distribution of halophytes across a salt marsh tidal gradient. Journal of Ecology, 2000, 88(4): 608-621.

[11] Xiao D R, Zhang L Q, Zhu Z C. The range expansion patterns of Spartina alterniflora on salt marshes in the Yangtze Estuary, China. Estuarine, Coastal and Shelf Science, 2010, 88 ( 1): 99- 104.

[12] Zhu Z C, Zhang L Q, Wang N, Schwarz C, Ysebaert T. Interactions between the range expansion of saltmarsh vegetation and hydrodynamic regimes in the Yangtze Estuary, China Estuarine, Coastal and Shelf Science, 2012, 96: 273-279.

[13] Liang X, Zhang L Q, Zhao G Q. A comparison of photosynthetic characteristics between Spartina alterniflora and Phragmites australis under different $\mathrm{CO} 2$ concentrations. Acta Ecologica Sinica, 2006, 26(3): 842-848.

[14] Huang H M, Zhang L Q, Guan Y J, Wang D H. A cellular automata model for population expansion of Spartina alterniflora at Jiuduansha Shoals, Shanghai, China. Estuarine, Coastal and Shelf Science, 2008, 77(1): 47-55.

[15] Li M, Yang S L, Li P, Liu Z, Dai S B, Gao A, Zhang J. Drastic decrease in sediment supply from Yangtze River and coastal crisis. Acta Geographica Sinica, 2006, 61 (3) : 282-288.

[16] Liu Y W, Yang S L, Luo X X. Separation estimate of beach erosion and deposition on intertidal wetland because of sea level rise and normal deposition. Shanghai Land and Resources, 2011, $32(3): 23-26$.

[17] Li H P, Zhang L Q, Wang D H. Distribution of an exotic plant Spartina alterniflora in Shanghai. Biodiversity Science, 2006, 14 (2) : 114-120.

[18] Schwarz C, Ysebaert T, Zhu Z C, Zhang L Q, Bouma T J, Herman P M J. Abiotic factors governing the establishment and expansion of two salt marsh plants in the Yangtze Estuary, China. Wetlands, 2011, 31(6) : 1011-1021.

[19] Thompson T L, Glenn E P. Plaster standards to measure water motion. Limnology and Oceanography, 1994, 39(7) : 1768-1779.

[20] Davis H G, Taylor C M, Civille J C, Strong D R. An Allee effect at the front of a plant invasion: Spartina in a Pacific estuary. Journal of Ecology, 2004, 92(2) : 321-327.
[21 ] Shen Y M, Yang J S, Wang Y H, Feng N H, Zhou Q, Zeng H. Impact of sediment supply on Spartina salt marshes. Pedosphere, $2008,18(5)$ : 593-598.

[22] He X Q, Dai X R, Liu Q Y, Li L J, Gu C J. Observation and analysis of the process of present-day morphology in the Chongming tidal flat of the Yangtze River estuary. Marine Geology \& Quaternary Geology, 2004, 24(2): 23-27.

[23 ] Yang S L, Xie W H, Zhu J, Zhao Q Y. A study of intertidal flat morphodynamics of a large river mouth: Yangtze River mouth. Geography and Territorial Research, 2001, 17(3): 4-8.

[24] Broome S W, Woodhouse W W, Seneca E D. Propagation of smooth cordgrass, Spartina alterniflora, from seed in North Carolina. Chesapeake Science, 1974, 15(4) : 214-221.

[25] Gascoigne J C, Beadman H A, Saurel C, Kaiser M J. Density dependence, spatial scale and patterning in sessile biota. Oecologia, 2005, 145(3): 371-381.

[26] Ysebaert T, Yang S L, Zhang L Q, He Q, Bouma T J, Herman P M J. Wave attenuation by two contrasting ecosystem engineering salt marsh macrophytes in the intertidal pioneer zone. Wetlands, $2011,31(6)$ : 1043-1054.

[27 ] van de Koppel J, van der Wal D, Bakker J P, Herman P M J. Self-Organization and vegetation collapse in salt marsh ecosystems. American Naturalist, 2005, 165(1) : E1-E12.

[28 ] Lambrinos J G, Bando K J. Habitat modification inhibits conspecific seedling recruitment in populations of an invasive ecosystem engineer. Biological Invasions, 2008, 10 ( 5 ): 729- 741 .

\section{参考文献:}

[13］梁霞, 张利权, 赵广琦. 芦苇与外来植物互花米草在不同 CO2 浓度下的光合特性比较. 生态学报. 2006, 26(3)：842-848.

[15］李明, 杨世伦, 李鹏, 刘哲, 戴仕宝, 郜昂, 张经. 长江来沙 锐减与海岸滩涂资源的危机. 地理学报, 2006, 61 (3): 282-288.

［16］刘英文, 杨世伦, 罗向欣. 海平面上升的淹没效应和岸滩冲淤 对潮间带湿地面积影响的分离估算. 上海国土资源, 2011, 32 (3) : 23-26.

[22] 何小勤, 戴雪荣, 刘清玉, 李良杰, 顾成军. 长江口崇明东滩 现代地貌过程实地观测与分析. 海洋地质与第四纪地质, $2004,24(2): 23-27$.

[23] 杨世伦, 谢文辉, 朱骏, 赵庆英. 大河口潮滩地貌动力过程的 研究一以长江口为例. 地理学与国土研究, 2001, 17(3): 4-8. 